\title{
Non-Destructive Testing of Mid-IR Optical Fiber Using Infrared Imaging
}

\author{
by M.-A. Gagnon*, V. Fortin**, R. Vallée**, P. Lagueux* and E. Guyot* \\ * Telops, 100-2600 Saint-Jean Baptiste, Québec, Qc, Canada, marc-andre.gagnon@telops.com \\ ${ }^{*}$ Centre d'optique photonique et laser, Université Laval, 2375 Terrasse, Québec, Qc, real.vallee@copl.ulaval.ca
}

\begin{abstract}
There is a growing need to develop high power mid-IR fiber lasers for a wide range of applications. However, the fluoride and chalcogenide glass fibers used for their fabrication must be exempt of defects in order to make efficient laser systems. To date however, most existing quality control techniques are not compatible with low energy optical band gap glasses such as chalcogenidesbecause of their limited transparency in the visible spectral range. This paper presents a novel non-destructive testing (NDT) methodology based on infrared imaging to address this problem. The results illustrate how this simple screening technique ease the selection of quality fibers for the design of high power mid-IR lasers.
\end{abstract}

\section{Introduction}

Many applications in the fields of medical surgery, polymer manufacturing, and defense and security [1] can benefit from the development of high-power fiber sources in the mid-infrared spectral range. These sources provides the advantage of being relatively compact, flexible and efficient, which allows their use in multiple configurations. Among the different materials compatible with mid-infrared $(2-25 \mu \mathrm{m})$ radiation are the fibers made with fluoride or chalcogenide glasses.

Mid-infrared lasers made of rare-earth doped fluoride glasses were previously shown to be very promising for biomedical applications (i.e. $2.8 \mu \mathrm{m}$ transition of erbium ions) [2]. Chalcogenide fibers were also used to make another type of mid-infrared laser based on stimulated Raman scattering [3]. The potential of chalcogenide fibers for high-resolution infrared imaging [4] and generation of mid-infrared supercontinuum [5], a broadband infrared source, was also reported. Despite their promising applications, manufacturing and/or synthesis of fluoride and chalcogenide glasses is still at a relatively early development stage. Consequently, the fibers often contain a non-negligible amount of defects, which affect their performances. In some cases, defects can be induced by gas bubbles emerging at the core-cladding interface. In addition, undesired micro-crystals are sometimes formed in the fiber core during the drawing process. Defects might lead to localized heating along the fiber, which can lead to material damage, power losses and even laser failure. For these reasons, quality control (QC) represents a very important aspect in the development of these technologies. However, many existing QC techniques are not compatible with chalcogenide fibers because of their limited transparency in the visible spectral range.

In this work, a non-destructive testing (NDT) methodology based on infrared imaging is developed to characterize the integrity of optical fiber. The experimental setup consists of an erbium doped fluoride fiber laser at $2.8 \mu \mathrm{m}$, optical fiber alignment stages and an InSb infrared camera (see Fig. 1). This camera allows the observation of laser scattering along the optical fibers, giving visual indication of defective areas. Comparison with images obtained with an added infrared spectral filter allows the identification of different types of defects (e.g. scattering vs absorbing defects). The results show how this simple screening technique eases the selection of high-quality fibers for the design of high-power mid-IR lasers.

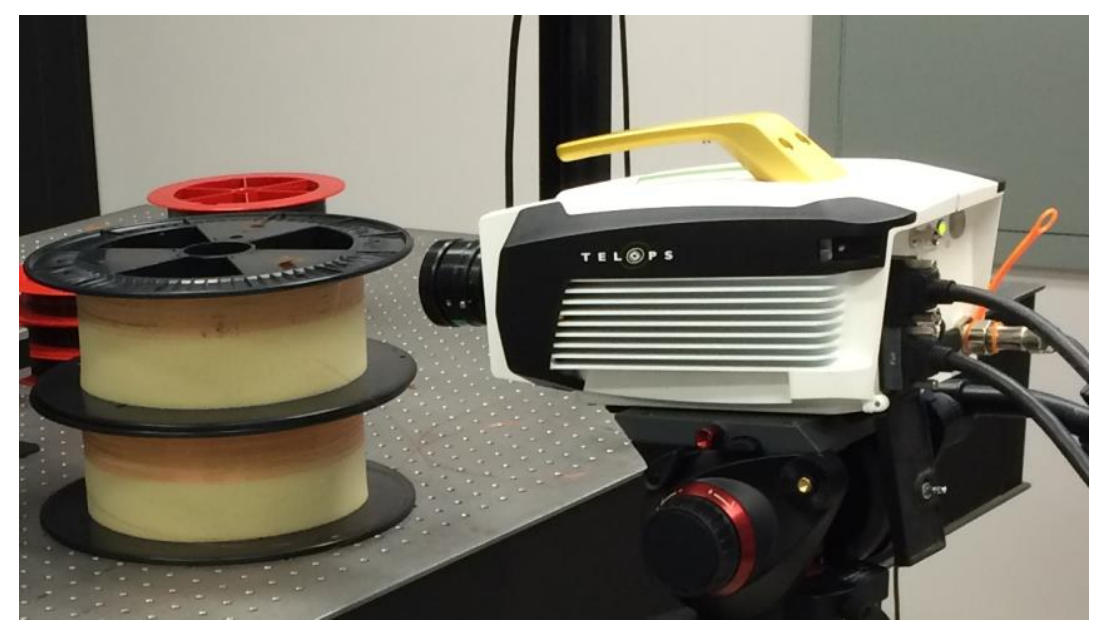

Fig. 1 Infrared imaging of an optical fiber bundle. 


\subsection{1/qirt.2016.036}

\section{Experimental Section}

\subsection{Fiber Laser}

The setup consists of a low-power $(100 \mathrm{~mW})$ in-house made fluoride glass fiber laser doped with erbium (see Fig. 2) operating at $2790 \mathrm{~nm}$, which is butt-coupled with the fiber spool to be tested [6]. A thermopile detector is placed at the end of the fiber to optimize the butt-coupling alignment and estimate propagation losses.

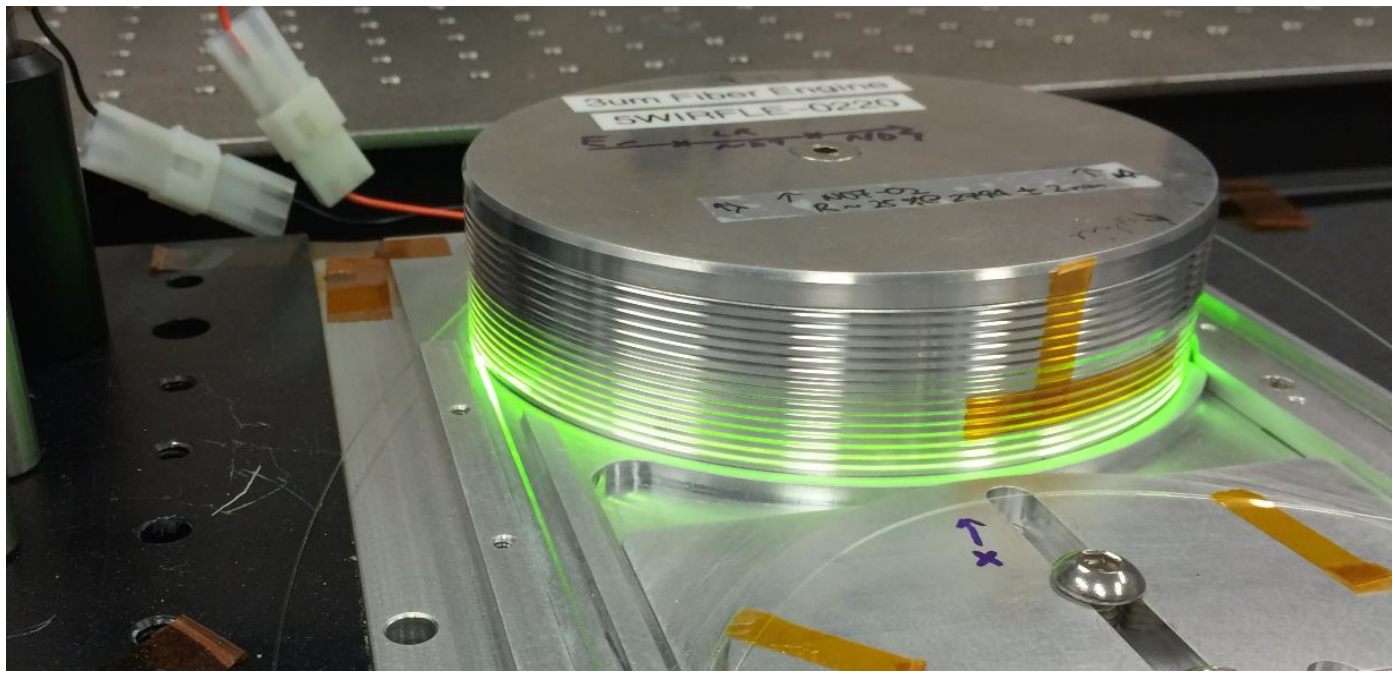

Fig. 2 Low-power Er-doped fluoride fiber laser at $2790 \mathrm{~nm}$.

\subsection{Infrared Imaging}

A Telops' midwave infrared $(1.5-5 \mu \mathrm{m})$ camera from the TEL-1000 series equipped with a $640 \times 512$ pixels indium antimonide (InSb) focal plane array (FPA) detector and a $25 \mathrm{~mm}$ lens was used for imaging. A long-pass $3.2 \mu \mathrm{m}$ spectral filter, placed in front of the lens, was used to record images without the laser's contribution. Images were recorded with the laser on and off, and with and without a spectral filter. In order to characterize the fibers at a higher spatial resolution without the laser's contribution, additional images were recorded using a Telops HD-IR (3.6 - $4.9 \mu \mathrm{m})$ camera equipped with a $1280 \times 1024$ pixel InSb detector as well as a $50 \mathrm{~mm}$ lens.

\section{Results and Discussion}

As shown in Fig. 3, a typical spool contains several tens of meters of fiber. Therefore, inspecting each sample millimetre by millimetre under the microscope is not a practical approach.

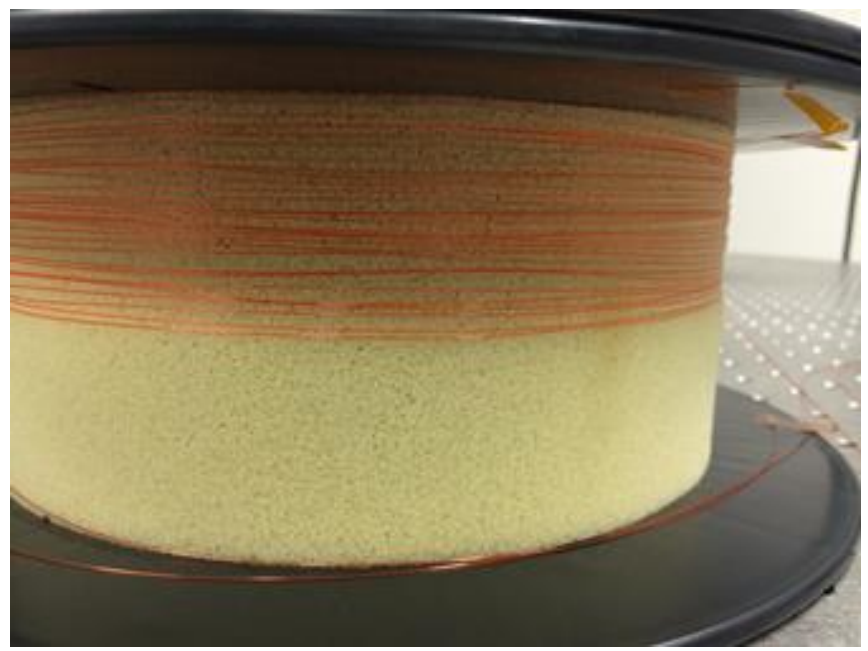

Fig. 3 Typical chalcogenide $\left(A s_{2} S_{3}\right)$ optical fiber spool to be tested. 


\subsection{1/qirt.2016.036}

For this reason, a non-destructive scanning technique providing information about the fiber integrity in a fast and efficient way represents an important asset in the development of fiber-based infrared lasers. There are many types of defects which can affect infrared radiation propagation within the fiber. The exact nature of these defects is still under investigation. Nonetheless, it is believed that some of them originate from the formation of micro-crystals in the core material and/or gas bubbles at the core-cladding interface during the drawing process. The refractive index change caused by their presence affect the course of the infrared radiation, as shown in Fig. 4. This results in radiation propagation within the core-cladding interstice, which can translate into localized heating and/or infrared scattering.

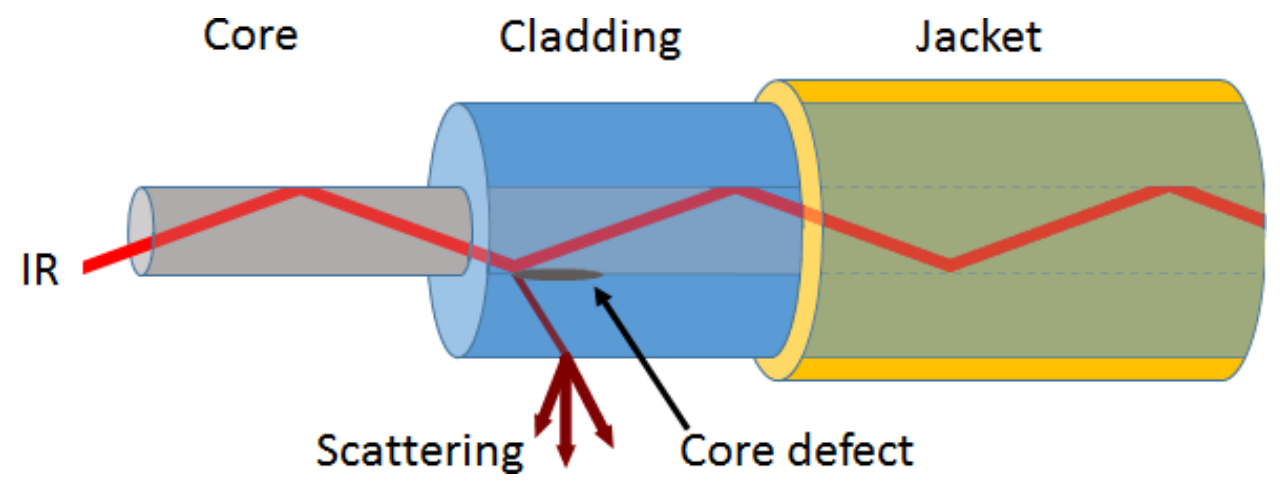

Fig. 4 Schematic representation of an infrared optical fiber.

A typical infrared image recorded on a chalcogenide fiber spool containing defects is shown in Fig. 5 (top). Bright spots associated with laser scattering can be seen along the fiber. According to Plank's blackbody curve, infrared selfemission is very weak (negligible) at $2.8 \mu \mathrm{m}$ under ambient conditions. Therefore, thermal contrasts measured in these conditions mostly originate from the laser. They come from localized heating but are dominated by laser scattering. By using a spectral filter that removes the $2.8 \mu \mathrm{m}$ laser's contribution, only the defects associated with localized thermal heating remain (see Fig. 5, bottom). Therefore, complementarity information is obtained by carrying out experiment with and without spectral filters to identify different defect types. 


\subsection{1/qirt.2016.036}
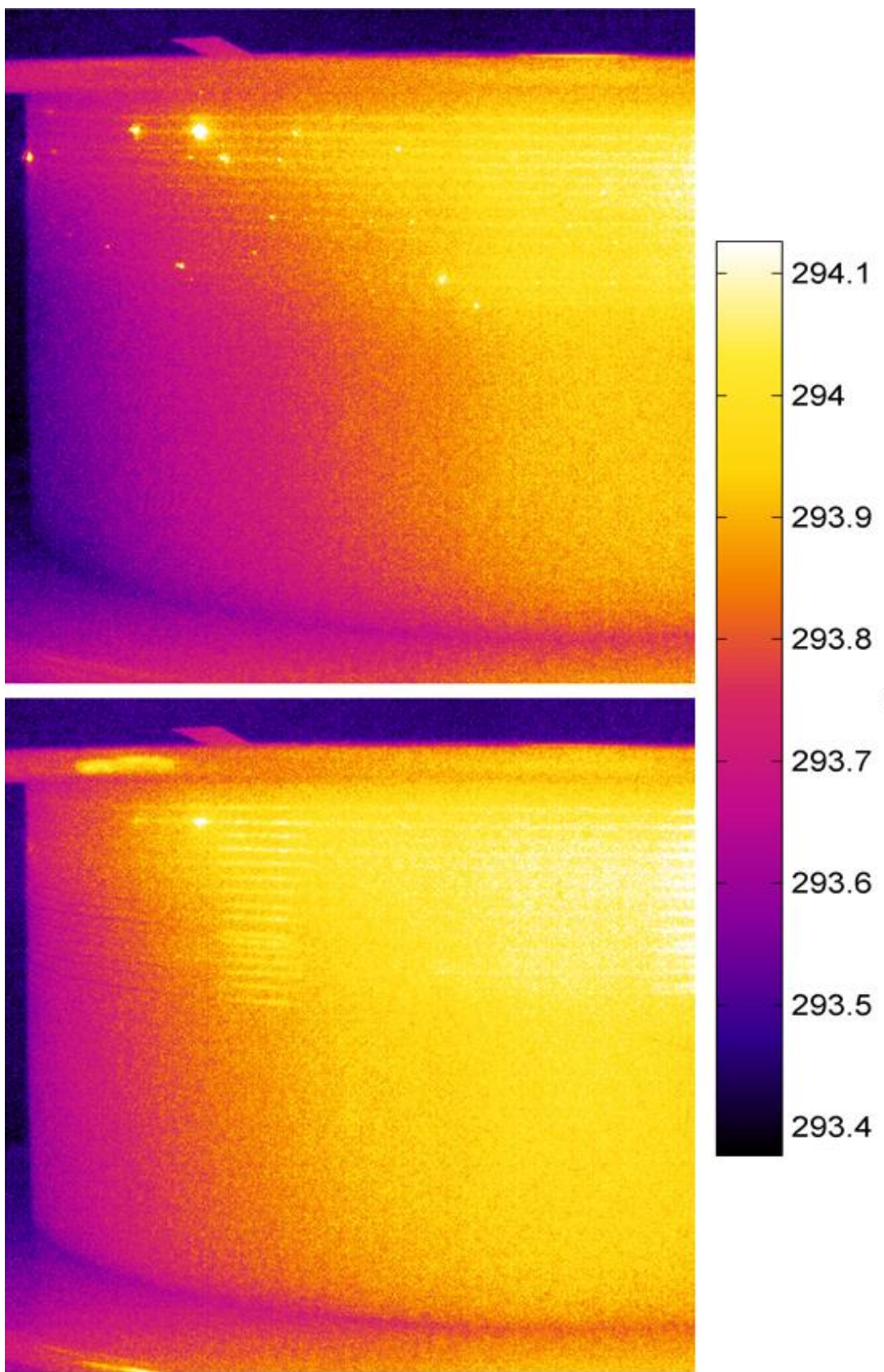

Fig. 5 Infrared imaging of a chalcogenide optical fiber bundle without (top) and with (bottom) a spectral filter.

By comparing the different images with a reference acquisition obtained with the laser source switched to off (image not shown), it is possible to sort the different types of defects within the sample, as shown in Fig. 6. Although the great majority of defects first seem to be co-localized (scattering + heating), there are some areas where only laser scattering can be measured. The defective areas are relatively small in size and only a few tens of degrees warmer than their surroundings. This makes them difficult to locate and emphasizes the need for high sensitivity. For this reason, additional measurements were carried out with a high-definition infrared camera, as shown in Fig. 7 . It should be noted that laser scattering cannot be observed using this specific infrared camera due of its narrower spectral range. Therefore, the images recorded under these conditions should be somewhat equivalent to the experiments carried out with the longpass spectral filter. Nevertheless, high-definition imaging $(1280 \times 1024)$ provides better spatial resolution as well as higher sensitivity compared with the experiment carried out with the TEL-1000 using an interferometric spectral filter at ambient temperature. The sensitivity requirement is highlighted in the temperature profile drawn across the defective area presented in Fig. 7. It can be seen that the temperature rises by only a few tens of degrees when a defective area is present along the fiber. 


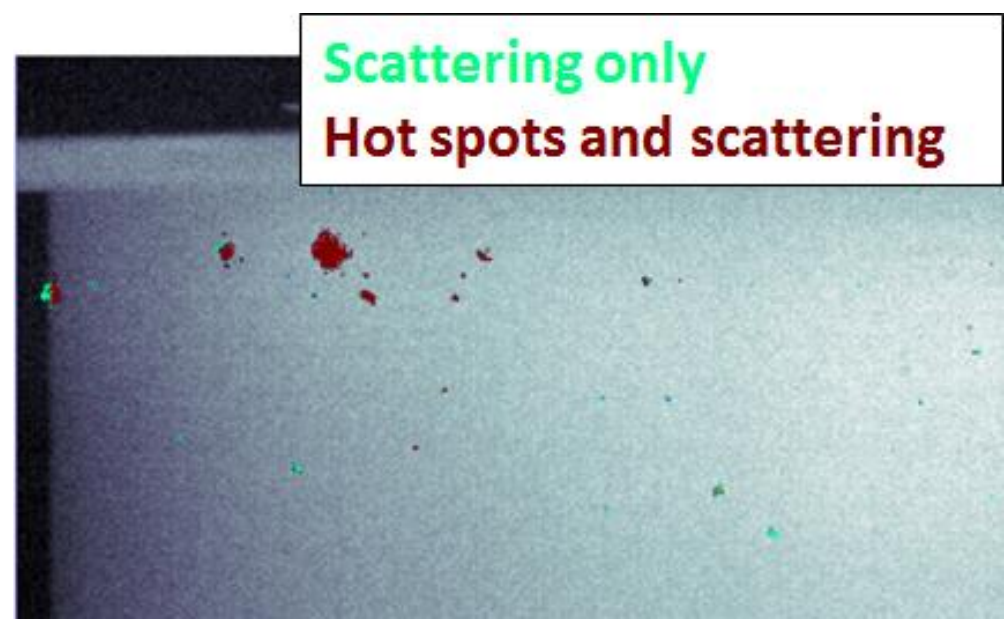

Fig. 6 Defect map of a chalcogenide optical fiber spool.
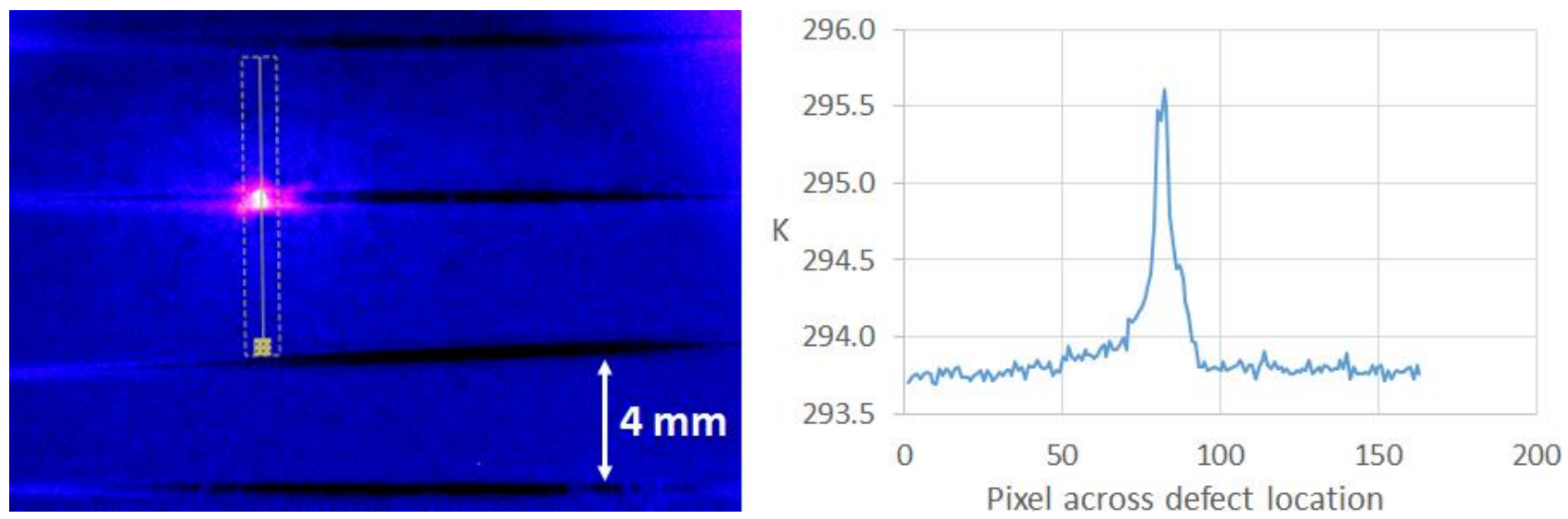

Fig. 7 High-resolution image of a defect using the HD-IR camera (left) and its temperature profile (right).

\section{Conclusion}

This work shows how infrared imaging can be used to carry out NDT of mid-IR optical fibers which are nontransparent in the visible spectral range. The methodology allows screening of tens of meters in just a few seconds. The complementary information obtained by comparing images recorded with and without a spectral filter allows the identification of different types of defects. Imaging carried out using the HD-IR camera provides both high spatial resolution and sensitivity requirements for NDT applications. The results show that imaging techniques can still be used for NDT of materials which are non-transparent in the visible spectral range.

\section{REFERENCES}

[1] Kim W.H., Nguyen V.Q., Shaw L.B., et al., Recent progress in chalcogenide fiber technology at NRL, Journal of Non-Crystalline Solids. - Vol. 431, 2882-2885 (2016).

[2] Serebryakoy V.A., Boǐko É.V., et al., Medical applications of mid-IR lasers. Problems and prospects, Laser Physics and Engineering. - Vol. 77, 6-17 (2010).

[3] Bernier M., Fortin V., El-Amraoui M., et al., $3.77 \mu \mathrm{m}$ fiber laser based on cascaded Raman gain in a chalcogenide glass fiber, Optics Letters. - Vol. 39, pp. 2052-2055, 2014.

[4] Zhang B., Zhai C., Qi S., et al., High-resolution chalcogenide fiber bundles for infrared imaging, Optics Letters. Vol. 40, pp. 4384-4387.

[5] Rosenberg C., Møller U., Kubat I., et al., Mid-infrared supercontinuum covering the 1.4-13.3 $\mu$ molecular fingerprint region using ultra-high NA chalcogenide step-index fibre, Nature Photonics. - Vol. 8, pp. 830-834, 2014.

[6] Fortin V., Bernier M., T. Bah S., et al., $30 \mathrm{~W}$ fluoride glass all-fiber laser at $2.94 \mu \mathrm{m}$, Optics Letters. - Vol. 40, pp. 2882-2885, 2015. 